ARTICLE

Received 26 Dec 2012 | Accepted 8 Feb 2013 | Published 12 Mar 2013 DOl: 10.1038/ncomms2593

\title{
Crystal structure of human tyrosylprotein sulfotransferase-2 reveals the mechanism of protein tyrosine sulfation reaction
}

Takamasa Teramoto1,2, Yukari Fujikawa1, Yoshirou Kawaguchi', Katsuhisa Kurogi ${ }^{3}$, Masayuki Soejima', Rumi Adachi ${ }^{1}$, Yuichi Nakanishi ${ }^{1}$, Emi Mishiro-Sato ${ }^{3}$, Ming-Cheh Liư ${ }^{4}$, Yoichi Sakakibara ${ }^{3}$, Masahito Suiko ${ }^{3}$, Makoto Kimura, ${ }^{1,2}$ \& Yoshimitsu Kakuta ${ }^{1,2}$

Post-translational protein modification by tyrosine sulfation has an important role in extracellular protein-protein interactions. The protein tyrosine sulfation reaction is catalysed by the Golgi enzyme called the tyrosylprotein sulfotransferase. To date, no crystal structure is available for tyrosylprotein sulfotransferase. Detailed mechanism of protein tyrosine sulfation reaction has thus remained unclear. Here we present the first crystal structure of the human tyrosylprotein sulfotransferase isoform 2 complexed with a substrate peptide (C4P5Y3) derived from complement $\mathrm{C} 4$ and $3^{\prime}$-phosphoadenosine- $5^{\prime}$-phosphate at $1.9 \AA$ resolution. Structural and complementary mutational analyses revealed the molecular basis for catalysis being an $\mathrm{S}_{\mathrm{N}} 2$-like in-line displacement mechanism. Tyrosylprotein sulfotransferase isoform 2 appeared to recognize the $\mathrm{C} 4$ peptide in a deep cleft by using a short parallel $\beta$-sheet type interaction, and the bound C4P5Y3 forms an L-shaped structure. Surprisingly, the mode of substrate peptide recognition observed in the tyrosylprotein sulfotransferase isoform 2 structure resembles that observed for the receptor type tyrosine kinases.

\footnotetext{
${ }^{1}$ Laboratory of Structural Biology, Graduate School of Systems Life Sciences, Kyushu University, Hakozaki 6-10-1, Fukuoka 812-8581, Japan. ${ }^{2}$ Laboratory of Biochemistry, Department of Bioscience and Biotechnology, Graduate School, Faculty of Agriculture, Kyushu University, Hakozaki 6-10-1, Fukuoka 812-8581, Japan. ${ }^{3}$ Food Research Branch, Department of Biochemistry and Applied Biosciences, Faculty of Agriculture, University of Miyazaki, Miyazaki 889-2192, Japan. ${ }^{4}$ Department of Pharmacology, College of Pharmacy and Pharmaceutical Sciences, The University of Toledo, Toledo, Ohio 43614, USA. Correspondence and requests for materials should be addressed to Y. Kakuta (email: kakuta@agr.kyushu-u.ac.jp).
} 
ost-translational protein modification by tyrosine sulfation, first discovered in bovine fibrinogen ${ }^{1}$, is now known to have a widespread occurrence among proteins in multicellular eukaryotic organisms ${ }^{2,3}$. While the functional involvement of protein tyrosine sulfation remains to be fully elucidated, it has been implicated in the alteration of biological activity of proteins, proteolytic processing of bioactive peptides ${ }^{4}$, change in half-life of proteins in circulation ${ }^{5}$, and modulation of extracellular protein-protein interactions including inflammatory leucocyte adhesion ${ }^{6,7}$. The recent discovery of the tyrosine sulfation of chemokine receptors suggests an even broader role in inflammatory response ${ }^{8,9}$. In the case of the chemokine receptor CCR5, the sulfated tyrosine residues located in its $\mathrm{N}$-terminal extracellular region have been shown to be crucial in mediating HIV binding/infection ${ }^{10-12}$.

Tyrosylprotein sulfotransferases (TPSTs) catalyse the transfer of a sulphonate moiety from $3^{\prime}$-phosphoadenosine- $5^{\prime}$-phosphosulfate (PAPS) to the hydroxyl group of protein-bound tyrosine residue to form a tyrosine $O$-sulphate ester and $3^{\prime}$-phosphoadenosine- $5^{\prime}$-phosphate (PAP) (Fig. 1a). TPSTs are integral membrane glycoproteins located in the trans-Golgi network $^{2}$. In humans, two TPST isoforms, designated TPST1 and TPST2, have been identified ${ }^{13-15}$. The two enzymes consist of 370 and 377 amino-acid residues, respectively, sharing $64 \%$ amino-acid sequence identity (Supplementary Fig. S1). They have been shown to display slightly different substrate specificities $^{16}$. Both TPST1 and TPST2 have the type II transmembrane topology with a short $\mathrm{N}$-terminal cytoplasmic domain, a single 17-residue transmembrane domain, and a putative stem region of about 40 residues, followed by a luminally oriented catalytic domain ${ }^{14,15}$.

Previous studies indicated that TPSTs recognize accessible tyrosine residues that are usually surrounded by several acidic residues within -5 to +5 positions ${ }^{17,18}$. However, no general consensus sequence for tyrosine sulfation sites has been defined. Furthermore, because no structure of TPST was available, the mechanisms by which the TPSTs recognize their protein substrates and catalyse the sulfation reaction had remained unclear.

Here we present the first crystal structure of the human TPST isoform 2 (TPST2) complexed with C4P5Y3, a substrate peptide derived from complement $\mathrm{C} 4$, and PAP at $1.9 \AA$ resolution. Structural and complementary mutational analyses revealed the molecular basis for both catalysis and substrate peptide recognition.

\section{Results}

Crystal structure of human TPST2. A crystal structure of a proteolytically defined $38-\mathrm{kDa}$ core domain of human TPST2 (designated TPST2 $\triangle \mathrm{C} 18$; encompassing Gly43-Leu359 of TPST2), complexed with PAP and C4P5Y3, was solved at a resolution of $1.9 \AA$. The $\mathrm{C} 4 \mathrm{P} 5 \mathrm{Y} 3$ peptide corresponds to a nine-amino-acid region encompassing the tyrosine sulfation site (Y741) of human complement C4 (Fig. 1b). This peptide, $\mathrm{E}^{-5}$ $\mathrm{D}^{-4}-\mathrm{F}^{-3}-\mathrm{E}^{-2}-\mathrm{D}^{-1}-\mathrm{Y}^{\text {acceptor }}-\mathrm{E}^{+1}-\mathrm{F}^{+2}-\mathrm{D}^{+3}$ (Yacceptor represents the acceptor site for sulfation), had been reported to be sulfated by the rat TPST with a $K_{\mathrm{m}}$ of $5.4 \mu \mathrm{M}$ (ref. 19). (Two Tyr residues (Y738 and Y743) in the original sequences have been mutated to Phe $(\mathrm{F})$ and only one Tyr (Y741) residue exists in the peptide.) The TPST $2 \Delta \mathrm{C} 18$ catalytic domain comprises a single $\alpha / \beta$ motif with a five-stranded parallel $\beta$-sheet, flanked on both sides by $\alpha$ helices (Fig. 2a). Central to this structural motif is the $5^{\prime}$-phosphosulfate-binding (5'-PSB) motif (75-GVPRSGTTL-83), contained within a strand-loop-helix consisted of $\beta 3$ and $\alpha 1$ (Supplementary Figs S2 and S3a). This loop can form extensive interactions with the $5^{\prime}$-phosphosulfate of PAPS. Moreover, $\beta 6$ and $\alpha 7$ are also key elements that include the $3^{\prime}$-phosphatebinding $\left(3^{\prime}-\mathrm{PB}\right)$ motif of PAPS (Supplementary Figs S2 and S3a). The $5^{\prime}$-PSB and $3^{\prime}$-PB motifs are known to be conserved among all members of the sulfotransferase family ${ }^{20,21}$. The arrangement of these secondary structures is very similar in structure to the P-loop found in some kinases such as UK as described in a previous report on SULT1E1 ${ }^{20}$.

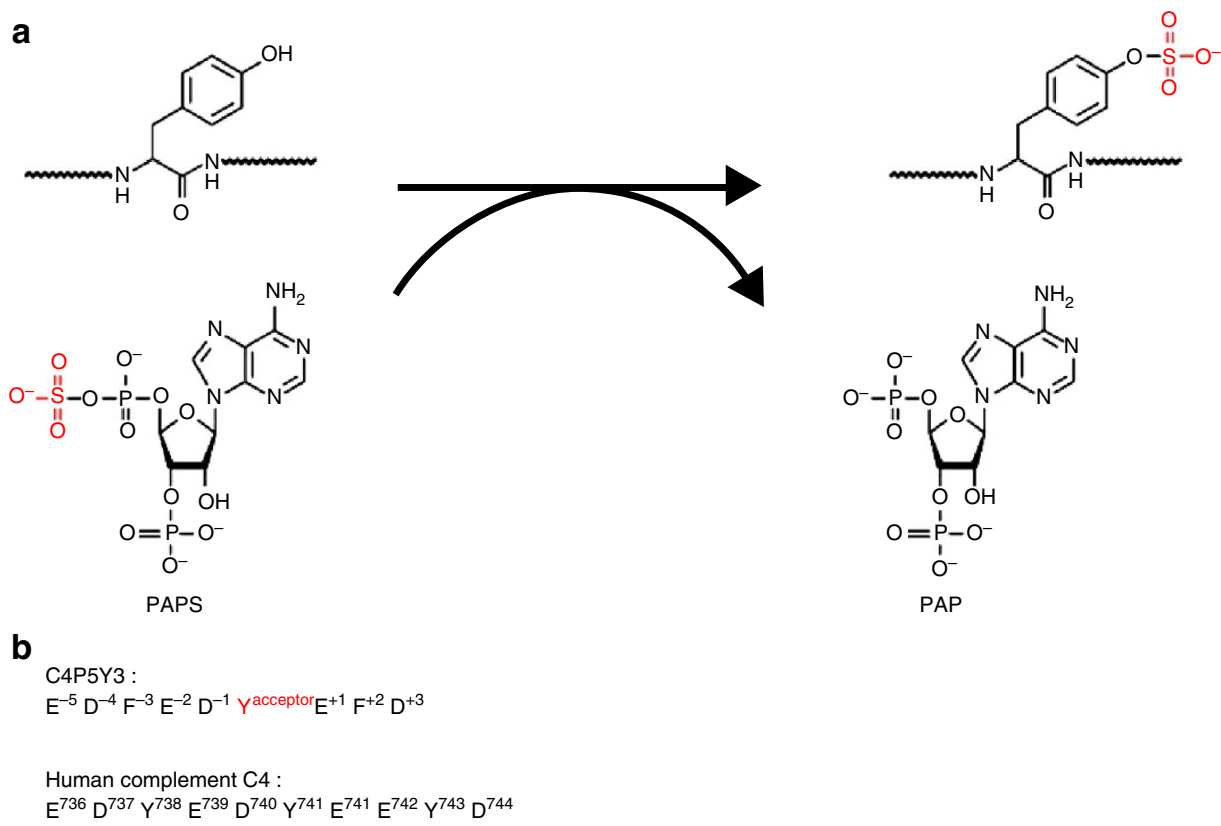

Figure 1 | Post-translational tyrosine sulfation reaction as catalysed by TPST. (a) The tyrosine residue of a substrate protein is sulfated by TPST using the sulphate donor, PAPS. (b) The amino-acid sequences of substrate peptides used in this study. The C4P5Y3 peptide is derived from a region encompassing a sulfation site $(Y 741)$ in human complement $C 4 . F^{-3}$ and $F^{+2}$ residues of $C 4 P 5 Y 3$ correspond to tyrosine residues ( $Y 738$ and $Y 743$, respectively) in complement $\mathrm{C} 4$ protein. 

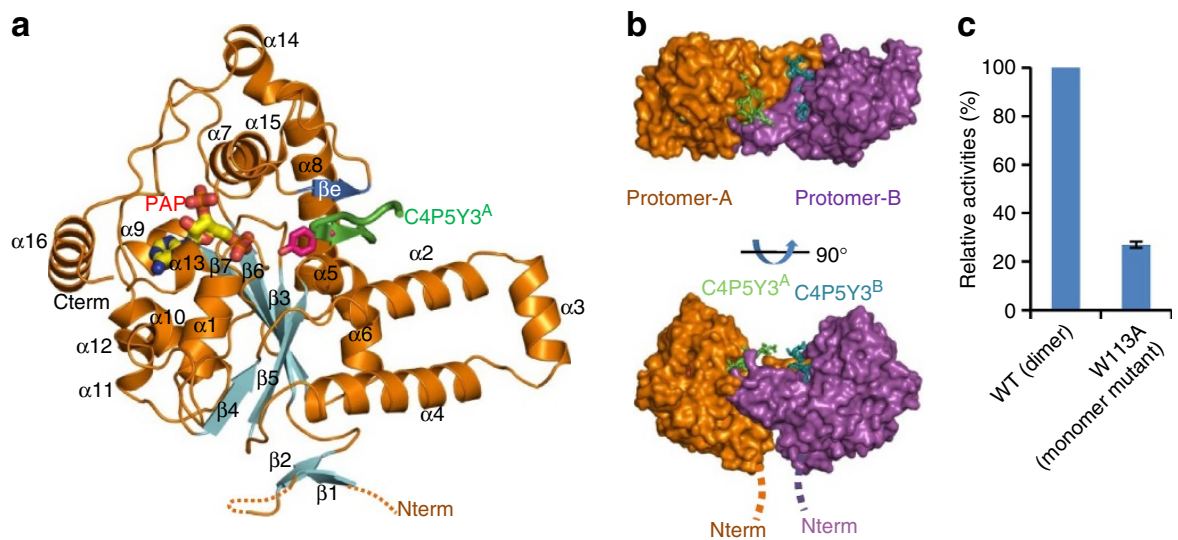

Figure 2 | The crystal structure of TPST2 $\Delta$ C18 in complex with PAP and C4P5Y3 peptide. (a) One protomer ribbon diagram of the TPST2 $\Delta C 18-P A P$ (yellow)-C4P5Y3 peptide (green) ternary complex. $\alpha$-helices and $\beta$-strands are coloured orange and cyan, respectively. The TPST2 $\Delta$ C18 catalytic domain comprises a single $\alpha / \beta$ motif with a five-stranded parallel $\beta$-sheet ( $\beta 7-\beta 6-\beta 3-\beta 5-\beta 4)$ flanked on both sides by $\alpha$-helices. Three consecutive $\alpha$-helices $(\alpha 2, \alpha 3$ and $\alpha 4)$ protrude from the roughly spherical configuration of the TPST2 $\Delta$ C18. (b) Dimeric complex of the core domain of TPST2 $\Delta C 18$. The lower diagram shows the side view of the dimer (protomer A and B are coloured orange and purple, respectively). The hypothetical positions of the $\mathrm{N}$ termini of both protomers have been sketched into the figure. The Golgi membrane is located ipsilateral to the $\mathrm{N}$-termini. The upper diagram shows the view of the TPST2 $\triangle \mathrm{C} 18$ dimer looking down the homodimer two-fold axis. The dimeric complex has two active sites and binds two C4P5Y 3 peptides. To distinguish between the two bound $\mathrm{C} 4 \mathrm{P} 5 \mathrm{Y} 3$ peptides, the peptides sulfated by A-protomer and B-protomer are referred to as $\mathrm{C} 4 \mathrm{P} 5 \mathrm{Y} 3^{\mathrm{A}}$ and $\mathrm{C} 4 \mathrm{P} 5 \mathrm{Y} 3^{\mathrm{B}}$, respectively. The diagrams shown in the figure were prepared using PyMol (http://pymol.sourceforge.net). (c) Site-directed mutagenesis study was performed to probe the role of W113 in dimer interface of TPST2. Sulfotransferase activity assay was performed using C4P5Y3 as the substrate peptide. The blue bar graph illustrates the relative activities of different mutant TPST2s, compared with that of the wild-type TPST2 as $100 \%$.

$n=3$, mean \pm s.e.m.

Dimer interface. Previous studies suggested that TPST enzymes exist as dimeric proteins that are formed primarily through the interaction of the transmembrane segments of the two constituent subunits ${ }^{15,22}$. Interestingly, although the transmembrane segment is not present, the $38-\mathrm{kDa}$ core domain of TPST $2 \Delta \mathrm{C} 18$ still forms a homodimer (with the two subunits designated, respectively, as protomers $\mathrm{A}$ and $\mathrm{B}$ ) as mediated by three consecutive $\alpha$-helices $(\alpha 2, \alpha 3$ and $\alpha 4$ ) (Fig. $2 \mathrm{~b}$ and Supplementary Fig. S3a). The dimer interface buries $\sim 25 \%$ of the surface area of each protomer. The orientation of the protomers positions the $\mathrm{N}$ termini of both subunits on one side of the dimer and in close proximity to each other (Fig. 2b). This orientation is consistent with a type II membrane-bound protein where both subunits would be anchored to the membrane via their $\mathrm{N}$-terminal transmembrane domains. That the TPST $2 \Delta \mathrm{C} 18$ core domain exists as a dimer was further supported by results from gel filtration chromatography (Supplementary Fig. S4a). TPST2 $\Delta \mathrm{C} 18$ was eluted with a calculated molecular mass of $\sim 77 \mathrm{kDa}$, consistent with that of a dimeric molecule. Additionally, a low-resolution TPST2 (Gly43-Ser377)-PAP binary complex structure without C4 peptides, belonging to a different space group $\left(P 22_{1} 2_{1}\right)$, also showed a similar dimeric structure (Supplementary Fig. S4b). The dimerization results in the formation of the acceptor-substrate binding sites in both protomers. Although no disulphide bond is present between the protomers in either of the two crystal structures, C31, which is not included in crystallized regions, is present immediately after transmenbrene domain. Therefore, it is possible that $\mathrm{C} 31$ could be involved in homodimer formation in the full-length enzyme.

The dimer formation mediated by the three consecutive $\alpha$-helices situates R118 and R122 in positions to participate in acceptor-substrate binding of the other protomer, and produces a wide positively charged surface (Supplementary Figs S3a and S5a). W133 exits in dimer interface on $\alpha 2$ and, interestingly, W133A mutant appeared to exist as monomer in solution based on the gel filtration chromatography result
(Supplementary Fig. S4a). Moreover, W113A mutant showed a significant decrease in sulfotransferase activity compared with the wild-type enzyme (Fig. 2c). These results indicate that while dimerization of TPST2 is not essential for catalysis, it does have some role in substrate binding.

Comparison with other sulfotransferases and catalytic mechanism of TPST2. As with TPST2, heparan sulphate sulfotransferases, including 3-O-sulfotransferase isoform 3 (HS3OST3) ${ }^{23}$, are type II transmembrane Golgi-associated enzymes. Cytosolic sulfotransferases (SULTs), for example, mouse SULT1D1 (ref. 24), differ from their Golgi-associated counterparts in that they are soluble enzymes and recognize lowmolecular-weight compounds, such as hydrophobic steroid and xenobiotics, as acceptor substrates. Structural comparisons of TPST2 $\triangle \mathrm{C} 18$ with HS3OST3 and SULT1D1 show a root mean squared deviation (r.m.s.d.) of $2.9 \AA$ for 167 structurally equivalent $C \alpha s$ and $2.8 \AA$ for 162 structurally equivalent Cas, respectively, but with only low sequence identities (19.1\% and $14.8 \%$, respectively). Particularly, the core elements including the $5^{\prime}$-PSB and $3^{\prime}$-PB motifs, which are involved in the binding to PAPS, are structurally conserved (Supplementary Fig. S3a-c).

Superposition of TPST2 $\Delta$ C18 with the active site of SULT1D1 complexed with PAPS and $p$-nitrophenol ( $p$ NP; yellow) and that of HS3OST3 complexed with PAP and tetrasaccharide (cyan) shows that residues involved in catalytic reaction are also structurally conserved. This superposition revealed the catalytic functions of residues R78, E99, K158 and S285 of TPST2 $\triangle$ C18 (Fig. 3a, Supplementary Fig. S3a). In the HS3OST3 structural study, E184 was proposed to serve as a catalytic base, deprotonating the 3-OH group of the glucosamine unit $(\mathrm{GlcN})$ of tetrasaccharide for nucleophilic attack on the sulphonate group. A structurally equivalent E99 is present in TPST2 $\Delta \mathrm{C} 18$. The mutation of this residue resulted in a complete loss of sulfotransferase activity for mutated TPST2 (Fig. 3b), suggesting 
a

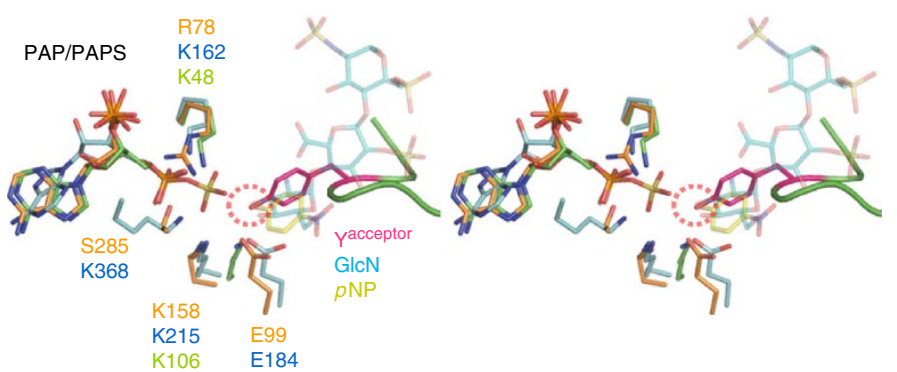

b

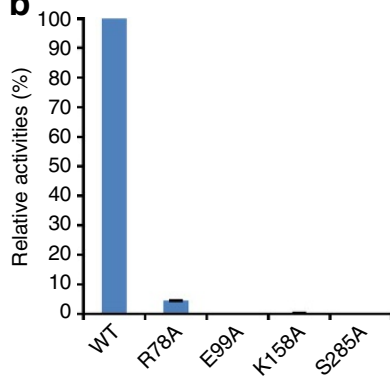

Figure 3 | Superposition of the catalytic sites of TPST2 $\mathbf{C}$ C18, SULT1D1 and HS30ST3. (a) Stereo figure of superposition of TPST2 $\Delta$ C18 with the

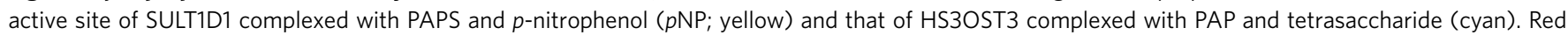
dashed circles indicate the positions of the three acceptor hydroxyl groups of substrates. (b) Site-directed mutagenesis experiments were performed to probe the role of specific amino-acid residues in the catalysis of TPST2. Sulfotransferase activity assay was performed using C4P5Y3 as the substrate peptide. The blue bar graph illustrates the relative activities of different mutant TPST2s, compared with that of the wild-type TPST2 as $100 \%$. $n=3$, mean \pm s.e.m.

that E99 is indeed critical for the catalytic function of TPST2. R78 and S285 contact the bridge oxygen of the leaving phosphate group of PAP in TPST $2 \Delta \mathrm{C} 18$ structure. R78 of TPST2 $\Delta$ C18 superimposes very well with K48 of SULT1D1 and K162 of HS3OST3, which were proposed to act as possible proton donors in catalysis and stabilize the transition state ${ }^{25}$. Interestingly, the R78A mutant displayed significantly reduced enzymatic activity (Fig. 3b). S285 of TPST2 $\Delta$ C18 is at the equivalent position as K368 of HS3OST3. The mutation of K368 nearly abolished sulfotransferase activity for the mutated HS3OST3. The corresponding lysine residue of the $N$-sulfotransferase domain of $N$-deacetylase $/ N$-sulfotransferase- 1 (ref. 26), and other heparan sulphate sulfotransferases, has been proposed to have a significant role in catalysis, but detail of its catalytic role is unknown. The S285A mutant abolishes sulfotransferase activity completely for the mutated TPST2 (Fig. 3b). Sasaki et al. ${ }^{27}$ reported that H266Q mutant of mouse TPST lost catalytic activity. H267, a corresponding resiude in hTPST2, interacts with backbone carbonyl oxygen of V289 of $\alpha 13$. The abovementioned S285 is present immediately before $\alpha 13$. Therefore, this latter interaction seems to contribute to placing S285 in its correct position for catalysis. K158 of TPST2 $\Delta \mathrm{C} 18$ superimposes with K106 of SULT1D1 and K215 of HS3OST3. The lysine residue at this position has been proposed to function in the stabilization of the transition state. Interestingly, K158A mutation significantly reduced the enzymatic activity for the mutated TPST2 (Fig. 3b). In addition, the positions of acceptor hydroxyl groups of all three substrates (red dashed circles in Fig. 3a) and the $5^{\prime}$-phosphates of PAP/PAPS molecules are also well superimposed (Fig. 3a). The hydroxyl group of $\mathrm{Y}^{\text {acceptor }}$ is located $2.6 \AA$ from the sulphur atom of PAPS in SULT1D1 structure and this hydroxyl group is in a proper position for inline nucleophilic attack as previously proposed for other sulfotransferases (Fig. 3a). Taken together, the transfer reaction of TPST has been proposed to proceed through an $\mathrm{S}_{\mathrm{N}} 2$-like inline displacement in a sequential mechanism, in which R78, E99, K158, S285 have critical roles in catalysis. R78 is a catalytic acid and E99 is a catalytic base. K158 and S285 are residues for stabilization of the transition state. Previously, a ping-pong mechanism using a His residue as a catalytic residue was proposed $^{28}$. However, our structural and mutational studies, plus the absence of a His residue in the active site of the TPST $2 \Delta \mathrm{C} 18$ structure, strongly support a sequential mechanism. Moreover, it has been reported that $\mathrm{Mn}^{2+}$ ion influenced the activity of TPST $^{16}$. However, although our crystallization conditions included $\mathrm{Mn}^{2+}$ ion, we were unable to identify its position in the structure.

The shape of acceptor-substrate binding site and the mode of acceptor-substrate binding for these three sulfotransferases are totally different (Supplementary Fig. S3a-c). However, the residues involved in the acceptor-substrate binding mainly lie in the regions following $5^{\prime}$-PBS and $3^{\prime}$-PB in all three structures (Supplementary Fig. S3a-c). SULT1D1 takes small phenolic compounds of substrate into its hydrophobic substrate-binding site using a gate-like structure formed by two phenylalanine resides (Supplementary Fig. S3b). In contrast, HS3OST3 uses a widely open groove for binding to the sugar chain of substrate compounds forming many hydrogen bonds (Supplementary Fig. S3c). Different from these two enzymes, TPST2 $\Delta \mathrm{C} 18$ utilizes a deep narrow cleft formed by the dimer interface in substrate binding (Supplementary Figs S3a and S5). The abovementioned comparison reveals that acceptor substrates were recognized differentially by the corresponding regions around PAPS-binding motifs in three different types of sulfotransferases.

Substrate peptide binding. In both protomers, the bound C4P5Y3 peptide adopts an L-shaped conformation (flexed to an 86-degree angle at -1 position; Fig. 4a and Supplementary Fig. S6). Although each protomer binds the C4P5Y3 peptide in a slightly different manner, the interaction pattern and the structure of the $\mathrm{E}^{-2}-\mathrm{D}^{-1}-\mathrm{Y}^{\text {acceptor }}-\mathrm{E}^{+1}$ region are virtually identical between $\mathrm{C} 4 \mathrm{P} 5 \mathrm{Y}^{\mathrm{A}}{ }^{\mathrm{A}}$ and $\mathrm{C} 4 \mathrm{P} 5 \mathrm{Y} 3^{\mathrm{B}}$. All conserved interactions with C4P5Y3 between protomer A and B are shown in Fig. $4 \mathrm{a}-\mathrm{c}$. The backbone of $\mathrm{Y}^{\text {acceptor }}$ is recognized by a loop following the $3^{\prime}-\mathrm{PB}$ motif (backbone of Thr198-Ala200) as a short parallel $\beta$-strand $(\beta e)$ (Fig. $4 \mathrm{a}-\mathrm{c})$. Additionally, backbone atoms at -2 to -1 position are recognized by side chains of R101 and T198. The results obtained with the T198A TPST2 mutant showing some decrease in sulfotransferase activity (Fig. 4d) suggested that the T198 recognizes the backbone at -1 position. Although R101A is a monomeric mutant (Supplementary Fig. S4a), the decrease in sulfotransferase activity of the R101A mutant was higher than that of another monomeric mutant W113A (Fig. 2c). These results indicate that the recognition of backbone carbonyl oxygens of $\mathrm{E}^{-2}$ and $\mathrm{D}^{-1}$ of C4P5Y3 by R101 has some role in substrate binding. The side chain of $\mathrm{E}^{-2}$ forms no interactions (Fig. 4b,c). This finding is consistent with previous studies suggesting that the residue at -2 position in substrate peptide interacts with TPST using the peptide backbone ${ }^{29,30}$. Previous 
a

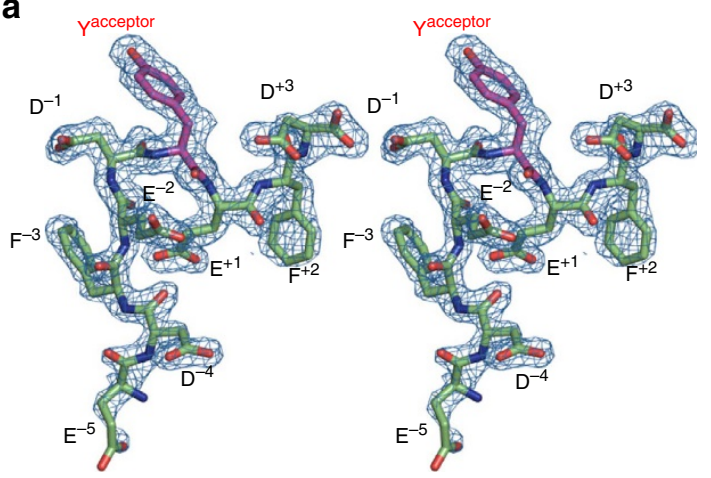

C

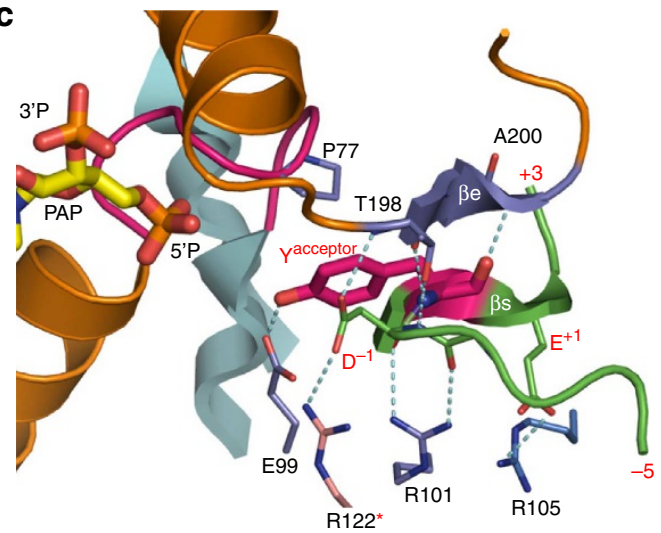

b

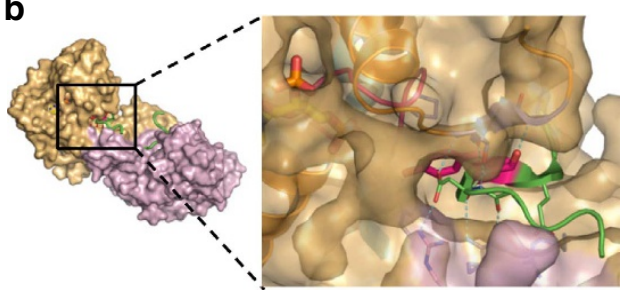

d

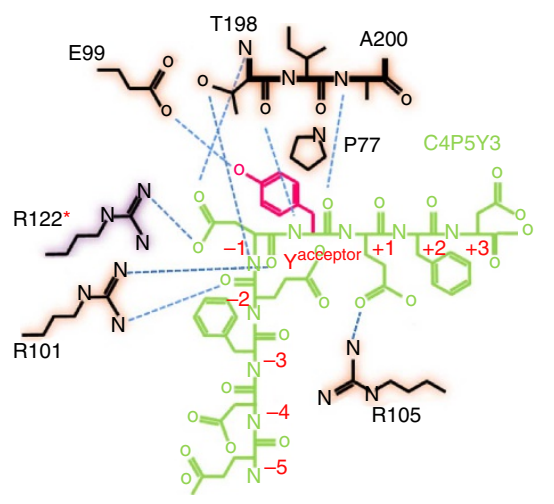

e

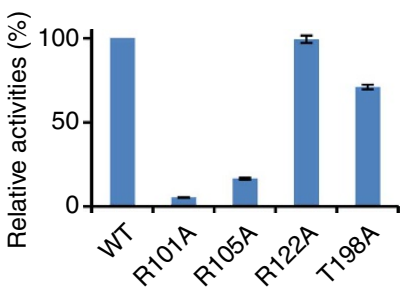

Figure 4 | Accepter peptide-binding site of TPST2 $\Delta$ C18. (a) The electron density maps showing the stereo image of bound C4P5Y $3^{A}$ peptide (simulated annealed omit Fo-Fc map contoured at $3 \sigma$ ). The acceptor tyrosine residue is coloured magenta. (b) Close-up views of the binding sites of $\mathrm{C} 4 \mathrm{P} 5 \mathrm{Y}^{\mathrm{A}}$ (green) with surface representation. (c) Close-up views of the binding sites of $\mathrm{C} 4 \mathrm{P} 5 \mathrm{Y} 3^{\mathrm{A}}$ (green). The interactions, in which the binding modes of TPST $2 \triangle \mathrm{C} 18$ for the peptides are identical between two active sites in the dimer, are shown. Residues of protomer $A$ that are involved in binding to $\mathrm{C} 4 \mathrm{P} 5 \mathrm{Y} 3^{\mathrm{A}}$ are coloured slate blue. R $122^{*}$ of protomer-B, which is involved in binding to $\mathrm{C} 4 \mathrm{P} 5 \mathrm{Y} 3^{\mathrm{A}}$, is coloured light pink. (d) Schematic representation of conserved interaction pattern of the TPST2 $\triangle$ C18 with C4P5Y3. (e) Site-directed mutagenesis experiments were performed to probe the role of specific amino-acid residues in the peptide binding of TPST2. Sulfotransferase activity assay was performed using C4P5Y 3 as the substrate peptide. The blue bar graph illustrates the relative activities of different mutant TPST2s, compared with that of the wild-type TPST2 as $100 \%$. $n=3$, mean \pm s.e.m.

studies showed, no general consensus sequence for tyrosine sulfation sites ${ }^{17,18}$. The substrate peptide backbone recognitions by TPST 2 as mentioned above seem to have an important role in substrate peptide binding.

The phenolic hydroxyl group of the $\mathrm{Y}^{\text {acceptor }}$ is hydrogen bonded to E99 and the aromatic ring forms a hydrophobic interaction with P77 of the 5'-PSB motif. A previous study indicated that the binding of TPST to a substrate peptide was enhanced in the presence of $\mathrm{PAP}^{13}$. This finding could be explained by the structural feature that the P77 is within the $5^{\prime}$-PSB motif and that $\beta e$ lies in the loop following the $3^{\prime}-\mathrm{PB}$ motif. Therefore, it can be postulated that PAP binding not only stabilizes $5^{\prime}$-PSB and $3^{\prime}$-PB regions but also forms the peptide-binding site.

Previous studies indicated that removal of the negative charge at -1 position of $\mathrm{C} 4 \mathrm{P} 5 \mathrm{Y} 3$ (cf. the $\mathrm{D}^{-1}$-to-N mutant) led to a 20 -fold increase in the $K_{\mathrm{m}}$ value $^{19}$. In the TPST $2 \Delta \mathrm{C} 18$ crystal structure, the $\mathrm{D}^{-1}$ of $\mathrm{C} 4 \mathrm{P} 5 \mathrm{Y} 3$ interacts with the side chain of $\mathrm{R} 122$ from the other protomer and the backbone amide nitrogen of T198 (Fig. 4b,c). The result obtained with the R122A TPST2 mutant showing little effect on the sulfotransferase activity (Fig. 4e) suggested that the negative charge of $\mathrm{D}^{-1}$ is specifically recognized by the backbone amide nitrogen of T198 (Fig. 4c,d).
The side chain of $\mathrm{E}^{+1}$ of C4P5Y3 is hydrogen bonded to R105 of TPST2. The results obtained with the R105A mutant showed a significant decrease in the sulfotransferase activity (Fig. 4d). These results indicate that the interaction contributes significantly to C4P5Y3 binding. However, as the negatively charged residue at +1 position of peptide substrates is not highly conserved, this side-chain interaction is likely not common for substrate peptide binding in TPST2.

The peptide-binding area is widely positively charged due to the presence of a number of arginine and lysine residues (R101, R105, K115, R118, R122, K164, K216 and R284; Supplementary Fig. S5a) and can thus accommodate negatively charged substrates that contain tyrosine residues adjacent to several acidic residues located within -5 to +5 positions. As described above, mutational analysis showed that only two residues (R101 and $\mathrm{R} 105)$ have some role in the recognition of C4P5Y3 (Fig. 4d) and all other residues are dispensable in terms of the recognition of C4P5Y3 (Supplementary Fig. S5b). It is possible, however, that these dispensable residues may have a role in the recognition of other protein substrates with acidic residues at the -5 to +5 position. It is to be pointed out that the original sequence of the C4P5Y3 peptide in complement $\mathrm{C} 4$ contains Tyr residues at the -3 and +2 positions. If $\mathrm{Tyr}$ 
residues are modelled back at these positions, the phenolic oxygen atoms in the -3 and +2 positions seem to form hydrogen bonds to R105 and R122, respectively. This latter finding indicates that Tyr residues in -5 to +5 position in the substrate sequence may contribute substrate recognition.

\section{Discussion}

TPSTs are enzymes responsible for the addition of a sulphonate group to tyrosine residues of target proteins during their posttranslational modification. Protein tyrosine sulfation has an important role in extracellular protein-protein interactions implicated in, for example, inflammation, haemostasis and viral infection. In this study, we solved the crystal structure of TPST2 $\Delta$ C18, a core domain of human TPST2, complexed with C4P5Y3, a peptide derived from complement C4, and PAP at $1.9 \AA$ resolution. Structural analysis indicated that TPST $2 \Delta \mathrm{C} 18$ is similar in terms of the catalytic mechanism to other types of sulfotransferases, but differ substantially in the substrate recognition mode.

This study demonstrated that TPST2 have four key features with regard to substrate peptide/protein recognition. First is that TPST2 can specifically recognize acceptor tyrosine on substrate proteins/peptides. This specific recognition is mediated by hydrogen bond interaction by Glu and hydrophobic interaction by Pro (Fig. 4c,d). Second is that TPST2 also recognizes backbone atoms of substrate proteins/peptides by two residues and $\beta$-sheet formation. The backbone atoms at -2 and -1 positions in the bound peptide are recognized by Arg and Thr of TPST2 and the backbone atoms of the acceptor tyrosine are recognized via a short parallel $\beta$-sheet type interaction (Fig. $4 \mathrm{c}, \mathrm{d}$ ). Third is that the bound substrate peptide binds in a deep cleft of TPST2 $2 \mathrm{C} 18$ and $\mathrm{Y}^{\text {acceptor }}$ on substrate peptide is placed deep in the active site of TPST2 (Fig. 4b). To access the deep active site, the vicinity of the accepter Tyr residue in the substrate proteins should probably take on an intrinsically unfolded conformation. Recently, Huang et al. ${ }^{31}$ reported that the coil structure contribute to sulfation site recognition. Thus, the majority of the sequences encompassing the accepter Tyr residue may be highly solvent-exposed and therefore are flexible and can easily adjust to the binding cleft of the TPSTs. Fourth is that TPST2 has many positively charged residues in its substrate-binding site and can recognize acidic residues within -5 to +5 position of the acceptor Tyr (Supplementary Fig. S5a). Taken together, an essential requirement for the substrate is that the acceptor tyrosine residue would have to be located in an intrinsically flexible region to fit into the deep cleft of hTPST2. Many electrostatic interactions are utilized in diverse substrate amino-acid sequences around tyrosine sulfation sites of proteins. Figure 5 shows a model for the sulfation of the soluble complement $\mathrm{C} 4$

Lumenal side of Golgi apparatus

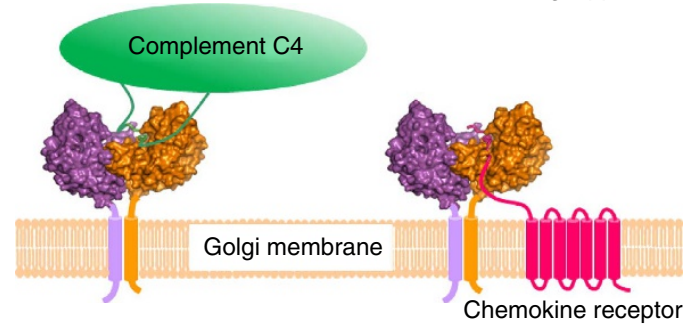

Figure 5 | Proposed model for the sulfation of soluble and membrane protein by TPST. The figure shows a schematic diagram illustrating the sulfation of the soluble complement C4 protein (green) and membranebound chemokine receptor protein (red) in the Golgi apparatus. protein and the membrane-bound chemokine receptor by hTPST2 in the Golgi lumen.

To further address the substrate recognition of hTPST2, we reviewed the correlation between substrate sequences and their $K_{\mathrm{m}}$ values. In a previous study, we determined kinetic constants for the TPST-mediated sulfation of various substrate peptides ${ }^{16}$. The tested peptide substrates can be divided into three groups based on their $K_{\mathrm{m}}$ values (low, moderately high and high). The first group (including complement C4, PSGL-1 and CCR2 peptides) with low $K_{\mathrm{m}}$ values contains $\mathrm{D}^{-1}$ and an acidic residue at +1 position (Supplementary Fig. S7). The second group (including CCR8 and CCK) with moderately high $K_{\mathrm{m}}$ values contain $\mathrm{D}^{-1}$, but no acidic resiude at +1 position (Supplementary Fig. S7). The third group (including CCR3 and CCR5) with high $K_{\mathrm{m}}$ values does not contain $\mathrm{D}^{-1}$ (Supplementary Fig. S7). The differences in $K_{\mathrm{m}}$ values can therefore be correlated with the existence of $\mathrm{D}^{-1}$ and an acidic resiude at +1 position. As already discussed in the TPST $2 \Delta \mathrm{C} 18$ crystal structure complexed with C4P5Y3, D ${ }^{-1}$ and $\mathrm{E}^{+1}$ in C4P5Y3 are specifically recognized by the backbone amide nitrogen of $\mathrm{T} 198$ and side chain of R105, respectively (Fig. 4c,d). Therefore, these two interactions are main determinants for the $K_{\mathrm{m}}$ values for substrate peptides. The conformations of $\mathrm{C} 4 \mathrm{P} 5 \mathrm{Y}^{\mathrm{A}}$ and $\mathrm{C} 4 \mathrm{P} 5 \mathrm{Y}^{\mathrm{B}}{ }^{\mathrm{B}}$ peptides are identical in the $\mathrm{E}^{-2}-\mathrm{D}^{-1}-\mathrm{Y}^{\text {acceptor }}-\mathrm{E}^{+1}$ region but divergent in the distal regions $\left(\mathrm{E}^{-5}-\mathrm{D}^{-4}-\mathrm{F}^{-3}\right.$ and $\mathrm{F}^{+2}-\mathrm{D}^{+3}$; Supplementary Fig. S6). The distal regions do not have conserved interactions between C4P5Y3 and protomer A or B (Fig. 4c). The side chains of $\mathrm{E}^{-5}$, $\mathrm{D}^{-4}, \mathrm{~F}^{-2}$ and $\mathrm{D}^{+3}$ in the peptides are exposed to solvent, indicating residues at these positions are unconstrained. In line with this postulation, residues at these positions have been shown to be unconserved ${ }^{31}$. For example, CCK peptide contains a positively charged Arg at -2 position and various residues at +3 position (Supplementary Fig. S7). To further elucidate the mechanism underlying substrate recognition by TPST, we will need to determine additional TPST structures complexed with other peptide substrates.

In humans, there are two isoforms of TPST (TPST1 and TPST2) and these two isoforms have slightly different substrate specificities $^{16}$. Comparison between human TPST1 and TPST2 showed that while the majority of the residues, which constitute the substrate-binding region are conserved (Supplementary Fig. S5c), several residues are not. These variations may therefore dictate the slight difference in substrate specificity between TPST1 and TPST2, and explain how they may accommodate a variety of substrate proteins.

A surprising, yet interesting, finding is that the mode of recognition for substrate peptide observed in the TPST $2 \Delta \mathrm{C} 18$ structure seems to resemble that observed for the receptor type tyrosine kinases (IRK) ${ }^{32}$ (Fig. 6), particularly in regard to the following three points: (i) $\beta$-sheet formation (parallel or antiparallel), (ii) hydrophobic interaction between phenol ring of target Tyr residue and Pro residue of the enzyme and (iii) hydrogen bond formation between phenolic oxygen of the target Tyr reside and acidic residue (Asp or Glu) of the enzyme. The intracellular locations of these two types of enzymes are different (Golgi lumen versus cytosol) and their substrate proteins also do not overlap. It is possible that the similarity between these two types of enzymes with regard to their peptide substrate recognition may be the result of convergent evolution, as they are both involved in the post-translational protein modification on Tyr residues.

To summarize, the molecular description of the TPST $2 \Delta \mathrm{C} 18$ crystal structure provides a framework for understanding the reaction mechanism of protein tyrosine sulfation, which is one of the major post-translational protein modifications. 

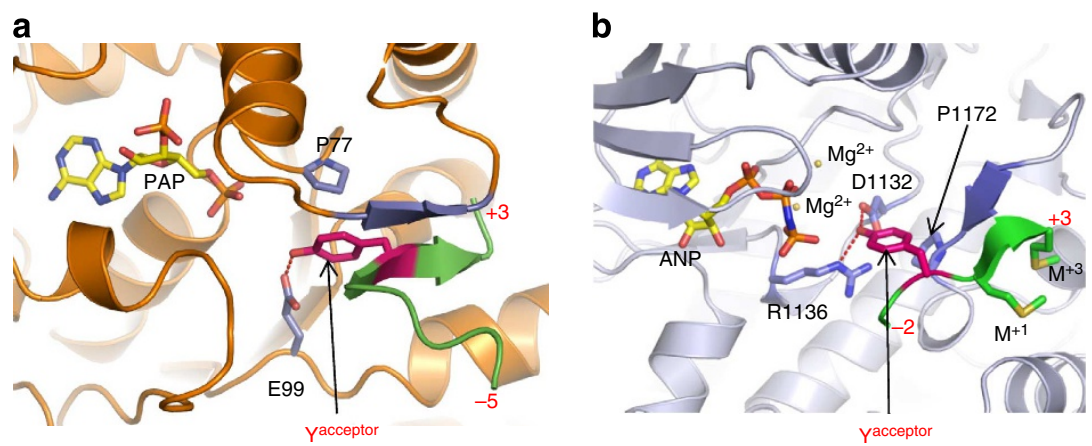

Figure 6 | Similarity between TPST2 and tyrosine kinase. (a) The active site of a ternary complex of TPST2 $\Delta$ C18. Yacceptor residue of the substrate peptide is coloured magenta and the rest is coloured green. The PAP is coloured yellow. Important residues for Yacceptor recognition are coloured slate blue. (b) The active site of a ternary complex of insulin receptor tyrosine kinase (IRK, PDB id code 1IR3). Yacceptor residue of the substrate peptide is coloured magenta and the rest is coloured green. The donor ATP analogue ANP is coloured yellow. Important residues for Yacceptor recognition are coloured slate blue.

\section{Methods}

Cloning, expression and purification. To circumvent potential complications associated with the crystallization of membrane proteins, we opted to express the proposed catalytic domain of TPST2 (Gly43-Ser377) that lacks the N-terminal tail, the transmembrane domain and part of the stem region. A complementary DNA (cDNA) encoding Gly43-Ser377 of TPST2 was PCR-amplified using a previously prepared pcDNA3-TPST-2 as the template ${ }^{16}$. The amplified cDNA was subcloned into pET15b (Novagen), which provided an $\mathrm{N}$-terminal 6xHis tag and a thrombin-recognition site. The plasmid construct (designated pET15b-TPST2) thus prepared was sequenced to confirm the reading frame and fidelity of the coding region using the service of the Research Support Center, Graduate School of Medical Sciences, Kyushu University. Recombinant protein was expressed in E. coli BL21 (DE3) RIL cells (Novagen) overnight at $37^{\circ} \mathrm{C}$. The cells were collected by centrifugation, resuspended in lysis buffer (containing $50 \mathrm{mM}$ Tris, $\mathrm{pH} 8.0$, and $500 \mathrm{mM} \mathrm{NaCl}$ ) and disrupted by sonication. The crude homogenate thus prepared was subjected to centrifugation at $12,000 \mathrm{~g}$ for $20 \mathrm{~min}$ and the pelleted fraction was resuspended in $1 \mathrm{M}$ glucose solution, followed by centrifugation at $12,000 \mathrm{~g}$ for $20 \mathrm{~min}$. The pellet was resuspended in $1 \%(\mathrm{v} / \mathrm{v})$ Triton X-100 solution containing $10 \mathrm{mM}$ EDTA, and centrifuged at $12,000 \mathrm{~g}$ for $20 \mathrm{~min}$. Afterwards, TPST2 was extracted from the pellet using an extraction buffer (containing $8 \mathrm{M}$ urea, $50 \mathrm{mM}$ Tris- $\mathrm{HCl}, \mathrm{pH} 8.0$, and $500 \mathrm{mM} \mathrm{NaCl}$ ) at $10^{\circ} \mathrm{C}$ for $1 \mathrm{~h}$. The extracted fraction was centrifuged at $12,000 \mathrm{~g}$ for $30 \mathrm{~min}$. The supernatant collected was applied to a Ni-NTA agarose gel (Qiagen) column, and the bound TPST2 was eluted from the column using the above-mentioned extraction buffer supplemented with $200 \mathrm{mM}$ imidazole. Refolding of the TPST2, thus purified, was performed based on a method modified from a previously reported slow dialysis protocol ${ }^{33}$. Afterwards, the buffer was exchanged with a binding buffer (containing $50 \mathrm{mM}$ Tris- $\mathrm{HCl}, \mathrm{pH}$ 7.0, and $100 \mathrm{mM} \mathrm{NaCl}$ ) using a Sephadex G-25 (GE Healthcare) buffer-exchange column. TPST2 thus prepared was further purified using a Resource S (GE Healthcare) cation-exchange column. Bound proteins were eluted using a linear gradient (from 0.1 to $1 \mathrm{M}$ ) of $\mathrm{NaCl}$, and the eluent was monitored by $\mathrm{A} 280 \mathrm{~nm}$. The purified soluble form of TPST2 was incubated with trypsin (Sigma) overnight at $10^{\circ} \mathrm{C}$, and the resulting proteolytically defined TPST2, a $38-\mathrm{kDa}$ core domain of TPST2 (TPST2 $\Delta 18$ ), was sequenced to show that it corresponds to the Gly43Leu359 region of TPST-2. A cDNA encoding Gly43-Leu359 (TPST2 $\Delta$ C18) was subcloned into pET15b (Novagen). The plasmid construct (designated pET15bTPST2 $\Delta C 18$ ) was sequenced to confirm the reading frame and fidelity of the coding region. Bacterial expression and purification of the recombinant TPST2 $\Delta$ C18 was performed using the same protocol for the recombinant TPST2 as described above. Purified TPST $2 \Delta \mathrm{C} 18$ was concentrated by ultrafiltration to a concentration of $2 \mathrm{mg} \mathrm{ml}^{-1}$. To the concentrated TPST2 $\Delta \mathrm{C} 18$ solution, PAP, $\mathrm{MnCl}_{2}$ and the C4P5Y3 peptide were added to final concentrations of 5, 1 and $1 \mathrm{mM}$, respectively.

Crystallization and data collection. Crystals of TPST2 $2 \mathrm{C} 18$ were prepared by using the sitting-drop vapour-diffusion method at $20^{\circ} \mathrm{C}$. The sitting drop contained $100 \mathrm{nl}$ of the above-mentioned TPST2 $\Delta \mathrm{C} 18$ preparation mixed with $100 \mathrm{nl}$ of a reservoir solution consisting of $0.1 \mathrm{M}$ sodium malonate ( $\mathrm{pH} 5.0$ ), and $10-12 \%$ polyethylene glycol 3350 . For data collection, crystals were transferred to a cryo buffer ( $0.1 \mathrm{M}$ sodium malonate, $\mathrm{pH} 5.0,10-12 \%$ polyethylene glycol 3350, 50 mM Tris-HCl, pH 7.0, $200 \mathrm{mM} \mathrm{NaCl}, 5 \mathrm{mM}$ PAP, $1 \mathrm{mM} \mathrm{MnCl}_{2}$, $5 \mathrm{mM}$ C4P5Y3 peptide and $10 \%$ glycerol). Thereafter, the preparation was flash frozen using a cryo system (Rigaku). A 1.9- $\AA$ resolution data set was collected at beamline BL5A, Photon Factory, Tsukuba, JAPAN. The structure of TPST2 $\Delta$ C18PAP-C4P5Y3 complex was solved by single isomorphous replacement and anomalous scattering using an iodide derivative. TPST2 $\triangle$ C18-PAP-C4P5Y3

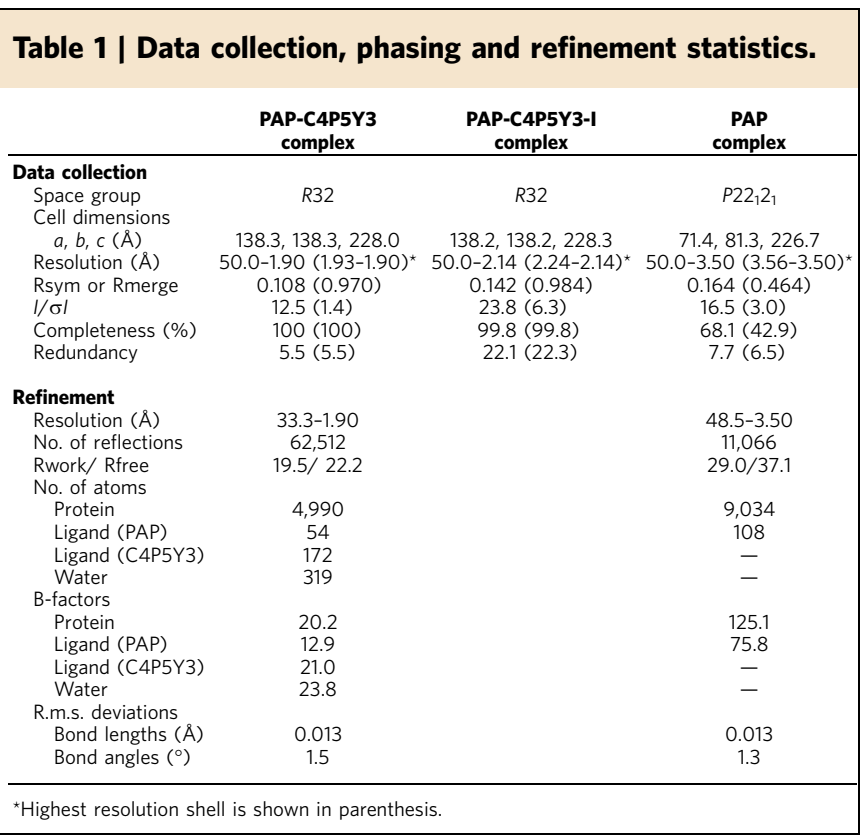

crystals were soaked for $30 \mathrm{~s}$ in a cryo buffer containing $1 \mathrm{M}$ potassium iodide and thereafter the preparation was flash frozen using a cryo system (Rigaku). A 22.1-fold redundant 2.0- $\AA$ data set was collected at beamline BL38B1, SPring-8, Hyogo, Japan.

For TPST2-PAP binary complex, PAP and $\mathrm{MnCl}_{2}$ were added to final concentrations of $5 \mathrm{mM}$ and $1 \mathrm{mM}$, respectively. Crystals of TPST2 were grown by using the sitting-drop vapour-diffusion method at $20^{\circ} \mathrm{C}$. The sitting drop contained $100 \mathrm{nl}$ of purified TPST2 solution mixed with $100 \mathrm{nl}$ of a reservoir solution consisting of $0.1 \mathrm{M}$ Tris- $\mathrm{HCl}, \mathrm{pH} 7.0,0.1 \mathrm{M}$ calcium acetate and $20 \%$ polyethylene glycol 3000 . For data collection, crystals were transferred to a solution containing $0.1 \mathrm{M}$ Tris- $\mathrm{HCl}, \mathrm{pH} 7.0,0.1 \mathrm{M}$ calcium acetate, $20 \%$ polyethylene glycol 3000, $50 \mathrm{mM}$ Tris-HCl, pH 7.0, $200 \mathrm{mM} \mathrm{NaCl}, 5 \mathrm{mM}$ PAP, $1 \mathrm{mM} \mathrm{MnCl} 2$ and $10 \%$ glycerol. Thereafter, the preparation was flash frozen in liquid nitrogen. A $3.5-\AA ̊$ resolution data set was collected at beamline BL17A, Photon Factory, Tsukuba, Japan.

Structure solution and refinement. The initial phase of the structure determination of the TPST2 $\triangle \mathrm{C} 18$-PAP-C4P5Y 3 complex was performed using the native and iodide derivative data with the program package HKL2MAP ${ }^{34}$. The density modification was applied to the initial phase using RESOLVE ${ }^{35}$. The partial model was built automatically using RESOLVE and ARP/wARP ${ }^{36}$, and modified manually using $\mathrm{COOT}^{37}$. The structure was revised several times by alternately adjusting the model and refinements using Refmac ${ }^{38}$. Refinement statistics are summarized in Table 1. Structure quality was assessed using PROCHECK ${ }^{39}$. 
The structure of PAP-bound form of TPST2 was solved by molecular replacement using the coordinates of TPST2 $\triangle \mathrm{C} 18$-PAP-C4P5Y3 dimer as a search model and the program Phaser $^{40}$ as a tool. The structures were revised several times by alternately adjusting the model and refinements using COOT and Refmac. Refinement statistics are summarized in Table 1. Structure quality was assessed using PROCHECK.

Mutational analysis. pET15b harbouring cDNAs encoding TPST2 mutants were prepared using the PCR-based Takara PrimeSTAR mutagenesis basal kit, with pET15b-TPST2 as the template, based on the protocol recommended by the manufacturer. The primers used for the PCR amplification of the mutants were synthesized by Sigma-Genosys. The PCR-amplified pET15b-TPST2 mutant constructs were sequenced to confirm the anticipated mutations using the service of the Research Support Cenetr, Graduate School of Medical Sciences, Kyushu University. TPST2 wild type and mutants for mutational analysis were expressed in Origami (DE3) cells (Novagen) carrying pGro7 (Takara, Japan) plasmids encoding chaperonin proteins GroEL and GroES. Upon an overnight induction at $30^{\circ} \mathrm{C}$, the cells were collected by centrifugation at $5,000 \mathrm{~g}$ for $20 \mathrm{~min}$, resuspended in lysis buffer and disrupted by sonication. The crude homogenate thus prepared was subjected to centrifugation at $12,000 \mathrm{~g}$ for $20 \mathrm{~min}$, and the supernatant was applied to a Ni-NTA agarose column. The bound proteins were eluted from the column using the lysis buffer containing $200 \mathrm{mM}$ imidazole. DTT (at the final concentration of $1 \mathrm{mM}$ ) was added to the eluted fraction, and the TPST2 therein was concentrated by centrifugal filters (Amicon Ultra 3K, Millipore), and further purified using a Superdex 200 16/60 (GE Healthcare) column. The eluted fractions containing TPST2 mutant protein were pooled. The buffer was exchanged with a new buffer (containing $50 \mathrm{mM}$ Tris-HCl, pH 7.0, and $150 \mathrm{mM}$ $\mathrm{NaCl}$ ) using a Sephadex G-25 buffer-exchange column. The purity of the TPST2 mutant protein thus prepared was confirmed by $12.5 \%$ SDS-PAGE. The sulfotransferse activity assays of wild-type and mutant TPST2s were performed as described previously ${ }^{16}$.

\section{References}

1. Bettelheim, F. R. Tyrosine-O-sulfate in a peptide from fibrinogen. J. Am. Chem. Soc. 76, 2838-2839 (1954)

2. Moore, K. L. The biology and enzymology of protein tyrosine O-sulfation. J. Biol. Chem. 278, 24243-24246 (2003).

3. Matsuzaki, Y., Ogawa-Ohnishi, M., Mori, A. \& Matsubayashi, Y. Secreted peptide signals required for maintenance of root stem cell niche in Arabidopsis. Science 329, 1065-1067 (2010).

4. Gregory, H., Hardy, P. M., Jones, D. S., Kenner, G. W. \& Sheppard, R. C. The antral hormone gastrin. Structure of gastrin. Nature 204, 931-933 (1964).

5. Leyte, A. et al. Sulfation of Tyr 1680 of human blood coagulation factor VIII is essential for the interaction of factor VIII with von Willebrand factor. J. Biol. Chem. 266, 740-746 (1991).

6. Pouyani, T. \& Seed, B. PSGL-1 recognition of P-selectin is controlled by a tyrosine sulfation consensus at the PSGL-1 amino terminus. Cell 83, 333-343 (1995).

7. Ippel, J. H. et al. Structure of the tyrosine-sulfated C5a receptor N terminus in complex with chemotaxis inhibitory protein of Staphylococcus aureus. J. Biol. Chem. 284, 12363-12372 (2009).

8. Choe, H. \& Farzan, M. Chapter 7. Tyrosine sulfation of HIV-1 coreceptors and other chemokine receptors. Methods Enzymol. 461, 147-170 (2009).

9. Veldkamp, C. T. et al. Structural basis of CXCR4 sulfotyrosine recognition by the chemokine SDF-1/CXCL12. Sci. Signal 1, ra4 (2008).

10. Farzan, M. et al. Tyrosine sulfation of the amino terminus of CCR5 facilitates HIV-1 entry. Cell 96, 667-676 (1999).

11. Cormier, E. G. et al. Specific interaction of CCR5 amino-terminal domain peptides containing sulfotyrosines with HIV-1 envelope glycoprotein gp120. Proc. Natl Acad. Sci. USA 97, 5762-5767 (2000).

12. Huang, C. C. et al. Structures of the CCR5 N terminus and of a tyrosinesulfated antibody with HIV-1 gp120 and CD4. Science 317, 1930-1934 (2007).

13. Niehrs, C. \& Huttner, W. B. Purification and characterization of tyrosylprotein sulfotransferase. EMBO J. 9, 35-42 (1990).

14. Ouyang, Y., Lane, W. S. \& Moore, K. L. Tyrosylprotein sulfotransferase: purification and molecular cloning of an enzyme that catalyzes tyrosine O-sulfation, a common posttranslational modification of eukaryotic proteins. Proc. Natl Acad. Sci. USA 95, 2896-2901 (1998).

15. Beisswanger, R. et al. Existence of distinct tyrosylprotein sulfotransferase genes: molecular characterization of tyrosylprotein sulfotransferase-2. Proc. Natl Acad. Sci. USA 95, 11134-11139 (1998).

16. Mishiro, E., Sakakibara, Y., Liu, M. C. \& Suiko, M. Differential enzymatic characteristics and tissue-specific expression of human TPST-1 and TPST-2. J. Biochem. 140, 731-737 (2006).

17. Hortin, G., Folz, R., Gordon, J. I. \& Strauss, A. W. Characterization of sites of tyrosine sulfation in proteins and criteria for predicting their occurrence. Biochem. Biophys. Res. Commun. 141, 326-333 (1986).
18. Rosenquist, G. L. \& Nicholas, Jr H. B. Analysis of sequence requirements for protein tyrosine sulfation. Protein Sci. 2, 215-222 (1993).

19. Lin, W. H., Larsen, K., Hortin, G. L. \& Roth, J. A. Recognition of substrates by tyrosylprotein sulfotransferase. Determination of affinity by acidic amino acids near the target sites. J. Biol. Chem. 267, 2876-2879 (1992).

20. Kakuta, Y., Pedersen, L. G., Carter, C. W., Negishi, M. \& Pedersen, L. C. Crystal structure of estrogen sulphotransferase. Nat. Struct. Biol. 4, 904-908 (1997).

21. Kakuta, Y., Pedersen, L. G., Pedersen, L. C. \& Negishi, M. Conserved structural motifs in the sulfotransferase family. Trends Biochem. Sci. 23, 129-130 (1998).

22. Goettsch, S. et al. Human TPST1 transmembrane domain triggers enzyme dimerisation and localisation to the Golgi compartment. J. Mol. Biol. 361, 436-449 (2006).

23. Moon, A. F. et al. Structural analysis of the sulfotransferase (3-o-sulfotransferase isoform 3) involved in the biosynthesis of an entry receptor for herpes simplex virus 1. J. Biol. Chem. 279, 45185-45193 (2004).

24. Teramoto, T. et al. Snapshot of a Michaelis complex in a sulfuryl transfer reaction: Crystal structure of a mouse sulfotransferase, mSULT1D1, complexed with donor substrate and accepter substrate. Biochem. Biophys. Res. Commun. 383, 83-87 (2009).

25. Negishi, M. et al. Structure and function of sulfotransferases. Arch. Biochem. Biophys. 390, 149-157 (2001).

26. Kakuta, Y., Sueyoshi, T., Negishi, M. \& Pedersen, L. C. Crystal structure of the sulfotransferase domain of human heparan sulfate $\mathrm{N}$-deacetylase/ N-sulfotransferase 1. J. Biol. Chem. 274, 10673-10676 (1999).

27. Sasaki, N. et al. A mutation in Tpst2 encoding tyrosylprotein sulfotransferase causes dwarfism associated with hypothyroidism. Mol. Endocrinol 21, 1713-1721 (2007).

28. Danan, L. M. et al. Catalytic mechanism of Golgi-resident human tyrosylprotein sulfotransferase-2: a mass spectrometry approach. J. Am. Soc. Mass Spectrom. 21, 1633-1642 (2010).

29. Bundgaard, J. R., Vuust, J. \& Rehfeld, J. F. New consensus features for tyrosine O-sulfation determined by mutational analysis. J. Biol. Chem. 272, 21700-21705 (1997).

30. Jen, C. H., Moore, K. L. \& Leary, J. A. Pattern and temporal sequence of sulfation of CCR5 N-terminal peptides by tyrosylprotein sulfotransferase-2: an assessment of the effects of N-terminal residues. Biochemistry 48, 5332-5338 (2009).

31. Huang, S. Y. et al. PredSulSite: prediction of protein tyrosine sulfation sites with multiple features and analysis. Anal. Biochem. 428, 16-23 (2012).

32. Hubbard, S. R. Crystal structure of the activated insulin receptor tyrosine kinase in complex with peptide substrate and ATP analog. EMBO J. 16, 5572-5581 (1997).

33. Maeda, Y., Koga, H., Yamada, H., Ueda, T. \& Imoto, T. Effective renaturation of reduced lysozyme by gentle removal of urea. Protein Eng. 8, 201-205 (1995).

34. Pape, T. \& Schneider, T. R. HKL2MAP: a graphical user interface for phasing with SHELX programs. J. Appl. Cryst. 37, 843-844 (2004).

35. Terwilliger, T. C. Maximum likelihood density modification. Acta Crystallogr. D Biol. Crystallogr. 56, 965-972 (2000).

36. Morris, R. J., Perrakis, A. \& Lamzin, V. S. ARP/wARP and automatic interpretation of protein electron density maps. Methods Enzymol. 374, 229-244 (2003).

37. Emsley, P. \& Cowtan, K. Coot: model-building tools for molecular graphics. Acta Crystallogr. D Biol. Crystallogr. 60, 2126-2132 (2004).

38. Murshudov, G. N., Vagin, A. A. \& Dodson, E. J. Refinement of macromolecular structures by the maximum-likelihood method. Acta Crystallogr. D Biol. Crystallogr. 53, 240-255 (1997).

39. Vaguine, A. A., Richelle, J. \& Wodak, S. J. SFCHECK: a unified set of procedures for evaluating the quality of macromolecular structure-factor data and their agreement with the atomic model. Acta Crystallogr. D Biol. Crystallogr. 55, 191-205 (1999).

40. McCoy, A. J. Solving structures of protein complexes by molecular replacement with Phaser. Acta Crystallogr. D Biol. Crystallogr. 63, 32-41 (2007).

\section{Acknowledgements}

This study was supported in part by a grant from the Research for Promoting Technological Seeds programme of Japan Science and Technology Agency (No. 15-050) and by funds from Kyushu University Interdisciplinary Programs in Education and Projects in Research Development. M.-C.L. was supported by an NIH grant (GM085756). We thank the staff of SPring-8 and the Photon Factory for their assistance in collecting data. We thank Drs Masa Negishi and Fritz Lipmann for their guidance of this work. The synchrotron radiation experiments were performed at the BL38B1 and BL44XU of SPring-8 with the approval of the Japan Synchrotron Radiation Research Institute (JASRI) (Proposal No. 2009B2096, 2010A1518 and 2010A6512), and Photon Factory (Proposal No. 2010G101).

\section{Author contributions}

T.T. performed experiments (protein production, crystallization, X-ray data collection and structure determination), analysed data, and prepared the manuscript; Y.F., 
Y. Kawaguchi, M. Soejima, R.A., Y.N. performed experiments (protein production, crystallization), analysed data; K.K., E.M.-S., Y.S. performed experiments (enzymological measurements), analysed data; M.-C.L., M. Suiko, M.K. analysed data and prepared the manuscript; Y. Kakuta devised the project, performed experiments (X-ray data collection and structure determination), analysed data, and wrote the paper.

\section{Additional information}

Accession codes: Structure factors TPST2 $\triangle \mathrm{C} 18-\mathrm{PAP}-\mathrm{C} 4 \mathrm{P} 5 \mathrm{Y} 3$ and TPST2-PAP have been deposited in the Protein Data Bank under accession numbers 3AP1 and 3AP3, respectively.
Supplementary Information accompanies this paper at http://www.nature.com/ naturecommunications

Competing financial interests: The authors declare no competing financial interests.

Reprints and permission information is available online at http://npg.nature.com/ reprintsandpermissions/

How to cite this article: Teramoto, T. et al. Crystal structure of human tyrosylprotein sulfotransferase-2 reveals the mechanism of protein tyrosine sulfation reaction. Nat. Commun. 4:1572 doi: 10.1038/ncomms2593 (2013). 